\title{
ACTIVIDADES DEL PROYECTO PERMAMODEL DURANTE LA CAMPAÑA ANTÁRTICA 2006-07
}

\author{
DAVID TOMÉ ${ }^{1}$ \\ Miguel RAMOS ${ }^{1}$ \\ Miguel Angel Hidalgo ${ }^{1}$ \\ GONÇALO VIEIRA ${ }^{2}$ \\ MÁRIo NEVES ${ }^{2}$ \\ AleXANDRE TRINDADE ${ }^{2}$
}

\section{INTRODUCCIÓN}

El estudio del papel del permafrost antártico como parte de la criosfera es abordado por dos de los proyectos del Año Polar Internacional: TSP - Thermal State of Permafrost (IPY $\mathrm{n}^{\circ}$ 50) y ANTPAS - Antarctic and Sub-Antartic Permafrost, Soils and Periglacial Environments (IPY $\mathrm{n}^{\mathrm{o}} 33$ ). La capa activa es muy sensible a la variabilidad climática, ya que corresponde a la capa superior de suelo sujeta a los procesos de congelación y descongelación anual en áreas con permafrost (Van Everdingen, 1998), siendo incluso afectado el propio permafrost (Ling e Zhang, 2004). Con la idea de estudiar estos efectos en emplazamientos de altas latitudes y zonas de montaña representativas de cada hemisferio y sensibles al cambio global se ha establecido la red CALM (Brown et al., 2000). Este protocolo se está implementando en Antártida con el nombre de CALM-S (Bockheim, 2004) y se incluye dentro de la red GTN-P, dedicado a la observación de la capa activa y de la situación térmica del permafrost subyacente (Burguess et al., 2001). El presente artículo desarrolla las experiencias y los resultados obtenidos durante la Campaña Antártica del 2006-08 por el proyecto PERMAMODEL.

\section{CARACTERIZACIÓN DE LA ZONA DE ESTUDIO}

Nuestra área de estudio, como se observa en la figura 1 se encuentra en las islas Livingston y Decepción, localizadas en el archipiélago antártico de las Shetlands del Sur a $62^{\circ} 39^{\prime} \mathrm{S}, 60^{\circ} 21^{\prime} \mathrm{W}$ y $62^{\circ} 43^{\prime} \mathrm{S}, 60^{\circ} 57^{\prime} \mathrm{W}$ en las cercanías de las Bases Antárticas Españolas (BAE) Juan Carlos I y Gabriel de Castilla, respectivamente.

Recebido: 03/04/2008. Revisto: 03/04/2009. Aceite: 01/06/2009.

1 Departamento de Física, Universidad de Alcalá, España. Email: david_tome@alu.uah.es, miguel.ramos@uah.es,miguel.hidalgo@uah.es

2 Centro de Estudos Geográficos, Universidade de Lisboa. Email: gtelesvieira@gmail.com, marioneves@netcabo.pt, alexandretn@gmail.com 

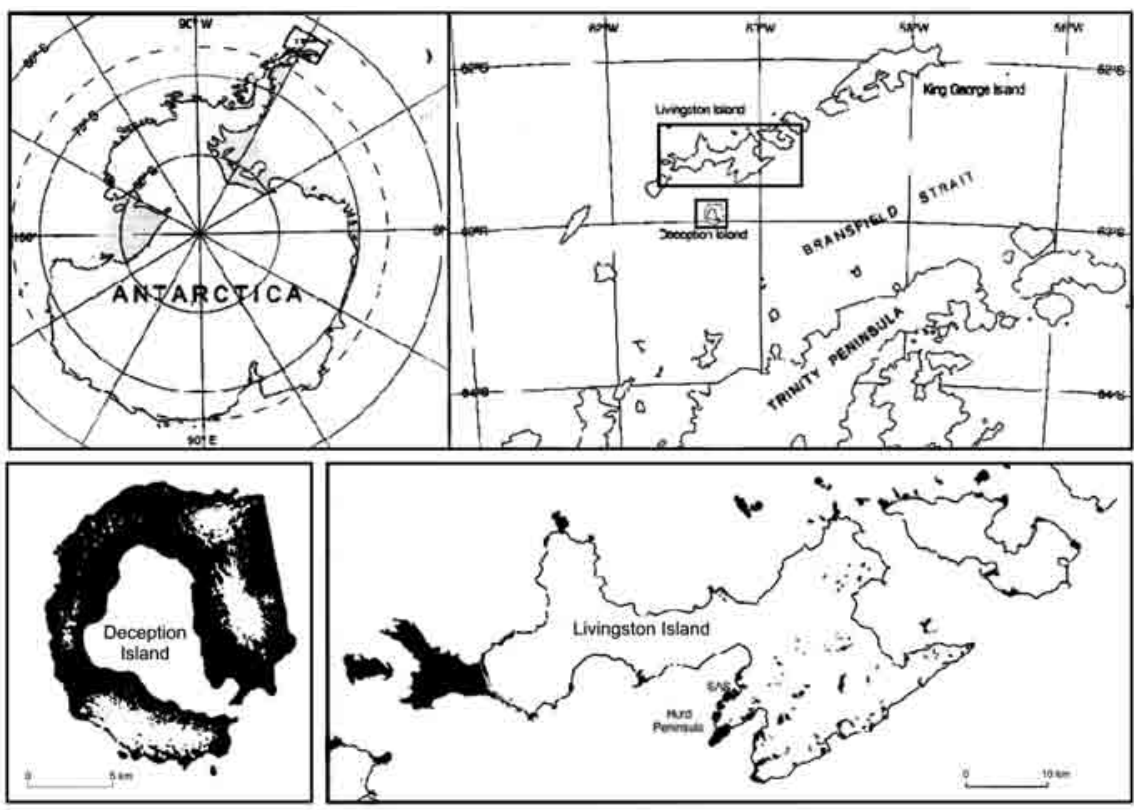

Fig. 1 - Localización del área de estudio (adaptado de López-Martinez et al., 1992). Fig. 1 - Location of the study area (adaptado de López-Martinez et al., 1992).

La BAE Juan Carlos I se sitúa en la costa sur de la península de Hurd en la isla Livingston. La isla tiene un $90 \%$ de territorio cubierto por hielo y nuestra área de trabajo se centra en las zonas no glaciadas cercanas a la BAE.

En el caso de Decepción, la isla es un estratovolcano activo en el que el flujo geotérmico influye sobre el comportamiento térmico del permafrost. El área de trabajo se encuentra cercana a la BAE Gabriel de Castilla y a Crater Lake.

La Península Antártica tiene una climatología con inviernos muy rigurosos con intensas precipitaciones y con temperaturas medias por debajo del punto de congelación del agua (King e Turner, 1997). El sistema de depresiones circumantártico condiciona esta situación. Las temperaturas medias en la Península Antártica van desde los $-5,2^{\circ} \mathrm{C}$ de Esperanza a los $-2,7^{\circ} \mathrm{C}$ ó $-1,6^{\circ} \mathrm{C}$ de Bellingshausen y Arctowski (Jones e Reid, 2001) y la humedad relativa presenta promedios entre 80 y $90 \%$ por la influencia marítima (Simonov, 1977; Styszynska, 2004).

\section{MÉTODO EXPERIMENTAL Y DATOS PRELIMINARES}

Uno de los objetivos de la campaña 2006-07 fue la instalación de tres estaciones de medida en Isla Livingston y otra en Isla Decepción, basadas en el protocolo CALM-S con el objeto de hacer un seguimiento de los parámetros físicos que afectan al comportamiento térmico del permafrost. Los detalles de estos emplazamientos se muestran en la Tabla I. 
Tabla I - Características de los emplazamientos CALM-S en las islas Decepción y Livingston Table I - Characteristics of the CALM-S sites in Deception and Livingston islands

\begin{tabular}{|c|c|c|c|c|c|c|c|}
\hline & $\begin{array}{c}\mathrm{N}^{\circ} \\
\text { Celdas }\end{array}$ & $\begin{array}{c}\text { Tamaño } \\
\text { de celda } \\
(\mathrm{m})\end{array}$ & $\begin{array}{l}\text { Posición } \\
\text { GPS }\end{array}$ & $\underset{\text { (m.s.n.m.) }}{\text { Altura }}$ & $\begin{array}{l}\text { Profundidad } \\
\text { de los } \\
\text { sondeos }(\mathrm{m})\end{array}$ & $\begin{array}{l}\text { Nivó- } \\
\text { metros }\end{array}$ & $\begin{array}{c}\text { Temperatura } \\
\text { ambiente }\end{array}$ \\
\hline $\begin{array}{l}\text { Crater Lake } \\
\text { (Decepción) }\end{array}$ & $10 \times 10$ & 10 & $\begin{array}{l}62^{\circ} 59^{\prime} 6^{\prime \prime} \mathrm{S}, \\
60^{\circ} 40^{\prime} 44^{\prime \prime} \mathrm{W}\end{array}$ & 112 & 1,7 y 2,0 & 2 & $\mathrm{Si}$ \\
\hline $\begin{array}{l}\text { Reina Sofía } \\
\text { (Livingston) }\end{array}$ & $4 \times 4$ & 10 & $\begin{array}{l}62^{\circ} 40^{\prime} 8^{\prime \prime} \mathrm{S}, \\
60^{\circ} 22^{\prime} 49^{\prime \prime} \mathrm{W}\end{array}$ & 270 & 1,3 & 1 & $\mathrm{Si}$ \\
\hline $\begin{array}{l}\text { Collado } \\
\text { Ramos } \\
\text { (Livingston) }\end{array}$ & $5 \times 5$ & 10 & $\begin{array}{c}62^{\circ} 40^{\prime} 2 ” \mathrm{~S} \\
60^{\circ} 23^{\prime} 31^{\prime \prime} \mathrm{W}\end{array}$ & 117 & 1,3 & 1 & $\mathrm{Si}$ \\
\hline $\begin{array}{l}\text { Ohridski } \\
\text { (Livingston) }\end{array}$ & $5 \times 5$ & 10 & $\begin{array}{l}62^{\circ} 38^{\prime} \mathrm{S} \\
60^{\circ} 21^{\prime} \mathrm{W}\end{array}$ & 140 & 0,4 & 2 & $\mathrm{Si}$ \\
\hline
\end{tabular}

Para conocer las variables que permiten caracterizar el balance energético de la capa activa en los emplazamientos CALM-S, se monitoriza la temperatura del aire, la temperatura del suelo en perforaciones de pequeña profundidad y el espesor de la capa de nieve. Éste último parámetro es clave, dado que la cubierta nival aísla térmicamente al suelo y este aislamiento afecta al intercambio de energía entre suelo y atmósfera (Zhang et al., 1996). Por esa razón los CALM-S están provistos de nivómetros basados en el registro de la evolución temporal de la temperatura en diferentes posiciones sobre la superficie del suelo en la capa limite superficial atmosférica. El análisis de estos registros en función de la inercia térmica observada nos permitirá estimar el espesor de dicha capa nival.

Por otro lado, se perforaron sondeos de pequeña profundidad $(1,3$ y $1,7 \mathrm{~m})$ con la intención de monitorizar el perfil de temperaturas de la capa activa durante todo el año. Estos sondeos son estancos, evitando el agua y la variación de humedad en el tubo. En su interior una varilla sustenta una serie de sensores de temperatura a diferentes profundidades, situándose la mayor parte de estos cerca de la superficie, donde se experimenta la mayor variabilidad térmica diaria y estacional. Las características de estas experiencias se muestran en las Tablas II, III y IV.

Tabla II - Instrumentación en CALM-S Crater Lake en Isla Decepción Table II - Monitoring equipment installed at the Crater Lake CALM-S site in Deception island

\begin{tabular}{|c|c|c|c|c|c|}
\hline & Sensores & Bits & $\begin{array}{l}\text { Periodo } \\
\text { (h) }\end{array}$ & $\begin{array}{c}\text { Posiciones } \\
\text { de los sensores }(\mathrm{cm})\end{array}$ & $\begin{array}{c}\text { Coordinadas } \\
\text { en la malla (m) }\end{array}$ \\
\hline Nivómetros & $\begin{array}{l}\text { i-Button } \\
1921 \mathrm{G}\end{array}$ & 8 & 4 & 2,$5 ; 5 ; 10 ; 20 ; 40$ & 30,30 y 70,70 \\
\hline Temperatura del Aire & Tiny Talk & 12 & 1 & 160 & 20,20 \\
\hline Temperatura del suelo & $\begin{array}{l}\text { i-Button } \\
\text { 1922L }\end{array}$ & 16 & 1 & $\begin{array}{c}-2,5 ;-5 ;-10 ;-20 ;-40 \\
-70 ;-100 ;-130\end{array}$ & 30,30 y 70,70 \\
\hline
\end{tabular}


Tabla III - Instrumentación instalada en los sitios CALM-S en Isla Livingston Table III - Monitoring equipment installed at the CALM-S sites in Livingston island

\begin{tabular}{|c|c|c|c|c|c|c|}
\hline & Localización & $\begin{array}{c}\text { Inicio del } \\
\text { registro }\end{array}$ & Sensores & Bits & $\begin{array}{l}\text { Periodo } \\
\text { (h) }\end{array}$ & $\begin{array}{c}\text { Posiciones } \\
\text { de los sensores }(\mathrm{cm})\end{array}$ \\
\hline \multirow[t]{2}{*}{ Nivómetros } & $\begin{array}{l}\text { Reina Sofía } \\
\text { Col. Ramos }\end{array}$ & 2006 & \multirow[t]{2}{*}{ TinyTalk } & \multirow[t]{2}{*}{12} & \multirow[t]{2}{*}{4} & $\begin{array}{c}2,5 ; 5 ; 15 ; 25 \\
50 ; 100\end{array}$ \\
\hline & Ohridski & 2007 & & & & 2,$5 ; 5 ; 10 ; 20 ; 40$ \\
\hline \multirow{2}{*}{$\begin{array}{l}\text { Temperatura } \\
\text { del Aire }\end{array}$} & $\begin{array}{l}\text { Col. Ramos } \\
\text { Reina Sofía }\end{array}$ & 2000 & \multirow[t]{2}{*}{ TinyTalk } & \multirow[t]{2}{*}{12} & 104 & \multirow[t]{2}{*}{160} \\
\hline & Ohridski & 2007 & & & 4 & \\
\hline \multirow{3}{*}{ Sondeos } & Col. Ramos & 2007 & $\begin{array}{l}\text { i-Button } \\
1922 \mathrm{~L}\end{array}$ & 16 & 1 & $\begin{array}{l}-2,5 ;-5 ;-10 ;-20 ;-40 ; \\
\quad-70 ;-100 ;-130\end{array}$ \\
\hline & Reina Sofía & 2000 & \multirow{2}{*}{ TinyTalk } & \multirow{2}{*}{12} & & $-5 ;-15 ;-40 ;-90$ \\
\hline & Ohridski & 2007 & & & 4 & $-5 ;-10 ;-20 ;-40$ \\
\hline
\end{tabular}

Tabla IV - Instrumentación instalada fuera de los sitios CALM-S en Isla Livingston Table IV - Monitoring equipment installed outside CALM-S sites in Livingston island

\begin{tabular}{llccccc}
\hline & Localización & $\begin{array}{c}\text { Inicio del } \\
\text { registro }\end{array}$ & Sensores & Bits & $\begin{array}{c}\text { Periodo } \\
\text { (h) }\end{array}$ & $\begin{array}{c}\text { Posiciones } \\
\text { de los sensores (cm) }\end{array}$ \\
\hline Nivómetros & Incinerador & 2006 & TinyTalk & 12 & 1 & $\begin{array}{c}2,5 ; 5 ; 15 ; 25 ; \\
50 ; 100\end{array}$ \\
Nivómetros & Nuevo incinerador & 2007 & $\begin{array}{c}\text { i-Button } \\
\text { 1921G }\end{array}$ & 8 & 4 & 2,$5 ; 5 ; 10 ;$ \\
& BAE Juan Carlos I & & & & & $20 ; 40$ \\
$\begin{array}{l}\text { Temperatura } \\
\text { del Aire }\end{array}$ & $\begin{array}{l}\text { Nuevo Incinerador } \\
\text { Refugio Motos }\end{array}$ & 2000 & TinyTalk & 12 & 1 & 160 \\
$\begin{array}{l}\text { Sondeos } \\
(24 \text { mm) }\end{array}$ & Nuevo incinerador & 2007 & $\begin{array}{c}\text { i-Button } \\
\text { 1922L }\end{array}$ & 16 & 1 & $-40 ;-70 ;-100 ;-130$ \\
$\begin{array}{l}\text { Sondeos } \\
(90 \mathrm{~mm})\end{array}$ & Incinerador & 2000 & TinyTalk & 16 & 1 & $-5 ;-15 ;-40 ;-90 ;$ \\
\hline
\end{tabular}

Dos tipos de sensores térmicos se han usado para las experiencias. Por un lado los Tiny talk con una resistencia NTC10k de $0,05^{\circ} \mathrm{C}$ de resolución y entre 0,1 a $0,2^{\circ} \mathrm{C}$ de precisión (Tiny Talk Gemini Co) y otros microsensores con $0,4^{\circ} \mathrm{C}$ de resolución, iButtons (Maxim Co.). Estas experiencias se complementan en cada nudo de la red CALM-S con el sondeo mecánico, hecho una vez cada año al final de verano, clavando una sonda de metal en el suelo hasta el permafrost, para medir el espesor máximo de la capa activa. Además, se hace también en cada nudo el sondeo térmico en superficie y a $15 \mathrm{~cm}$ de profundidad. 


\section{Actividades en Isla Decepción y resultados}

En la campaña 2007-08, en Isla Decepción se terminó de establecer el emplazamiento CALM-S que fue delimitado en la anterior campaña. Éste se encuentra sobre un terreno llano de ceniza y escombros volcánicos. Aunque muy poroso, el suelo tiene un alto contenido de agua intersticial. Se acompañó de dos nivómetros y sondeos, así como un sensor de temperatura ambiente. En la Tabla II mostramos las principales características de los sensores, así como su posición.

Además se realizaron sondeos mecánicos de capa activa en los vértices del CALM-S en una malla de 10x10m. Al compararse los resultados con los del año 2006 se observa que el espesor medio medio de capa activa es de $35,2 \mathrm{~cm}$ y para el año 2007 de $32 \mathrm{~cm}$ (fig. 2).

12 February 2006

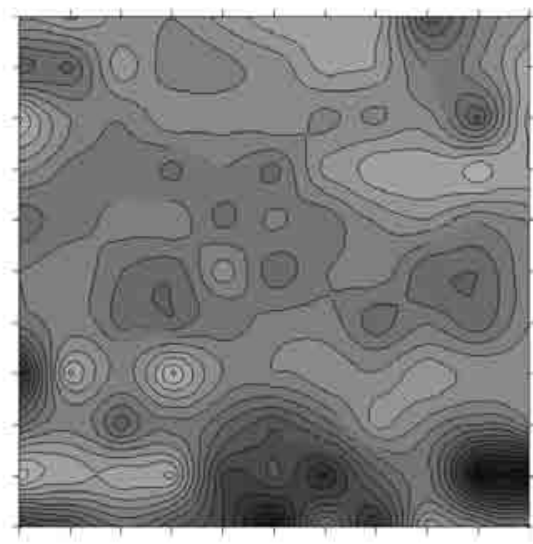

\section{January 2007}
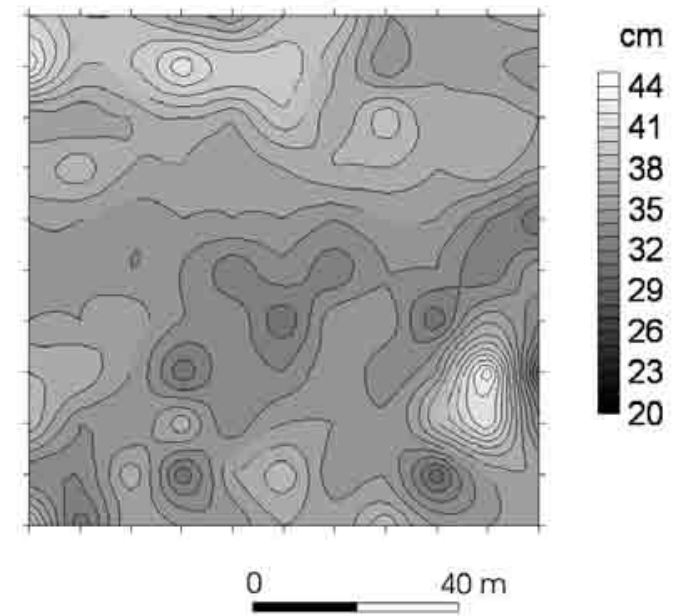

Fig. 2 - Espesor de la capa activa en CALM-S Crater Lake.

Fig. 2 - Active layer thickness at the Crater Lake CALM-S site.

Dos termómetros situados a $15 \mathrm{~cm}$ de profundidad en dos de los vértices del sitio CALM-S demuestran que el comportamiento térmico en el área es bastante homogéneo (fig. 3). 


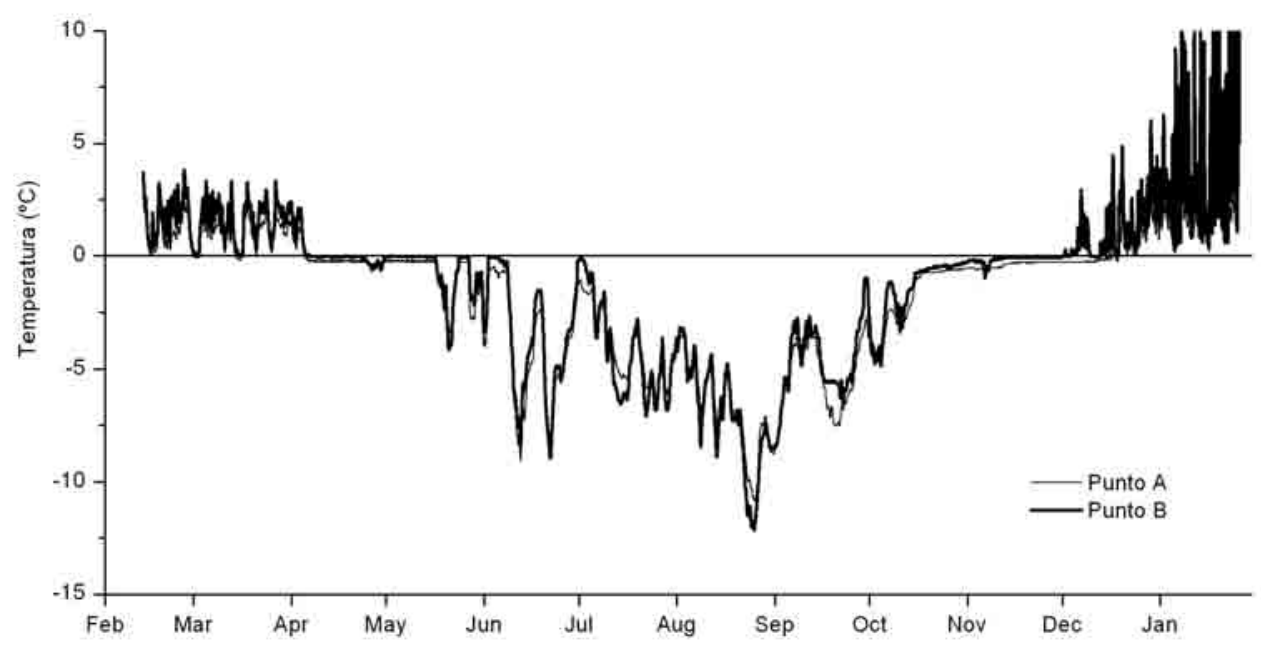

Fig. 3 - Evolución de las temperaturas a $15 \mathrm{~cm}$ de profundidad en los vértices del CALM Crater Lake en 2006.

Fig. 3 - Ground temperatures at $15 \mathrm{~cm}$ at two opposite corners of Crater Lake CALM-S site in 2006.

La temperatura del aire se mide con una sonda externa bajo un protector de radiación, para que la temperatura obtenida no sea influenciada por la radiación solar directa y a $1,60 \mathrm{~m}$ de altura.

Se instaló también en Punta Murature, un punto de medida TMEM (Transverse MicroErosion Meter) destinado a determinar la microerosión de la plataforma costera. Se realizó la primera toma de medidas, que en posteriores años someteremos a comparación.

\section{Actividades en Isla Livingston y resultados preliminares}

Durante la campaña del 2007-08 se establecieron tres asentamientos CALM-S en Isla Livingston: en la cima del pico Reina Sofía, en Collado Ramos y en la vecindad de la Base Búlgara St. Kliment Ohridski (Tabla I). Todos cuentan con un conjunto de instrumentación meteorológica que monitoriza la temperatura del aire, el espesor de la capa de nieve y el perfil térmico de la capa activa. Además de los sitios CALM-S, se ha instalado también instrumentación similar en otros puntos de la Península de Hurd, como muestra la Tabla III. Los emplazamientos CALM-S de la isla Livingston presentan unas dimensiones inferiores respecto a Decepción, dada la topografía más irregular y con superficies llanas mas limitadas. Por otro lado, sus suelos compuestos de rocas muy fragmentadas por los procesos periglaciares hace impracticable la medición mecánica del espesor de la capa activa. Las mediciones de temperatura de superficie y a $10 \mathrm{~cm}$ de profundidad se realizaron sin ningún problema. 
Se intentaron instalar nuevos sondeos en el nuevo CALM-S de Collado Ramos y de Reina Sofía. En el Collado Ramos se consiguió un sondeo de 1,3 m, pero en el Pico Reina Sofía, no se tuvo éxito, dado que el nivel freático se encontraba a $47 \mathrm{~cm}$ de profundidad. Además se instaló un nivómetro, una perforación con sensores de temperatura y una sonda de temperatura ambiente en la base de la ladera del sitio incinerador, en las cercanías de la Base Antártica Española, con el objetivo de comenzar la monitorización sobre un terreno llano, compuesto por materiales finos de arrastre por el agua de deshielo. Este emplazamiento fue designado como nuevo incinerador.

Desde el año 2000 se mantiene el registro de los datos correspondientes a los sondeos de Incinerador y Reina Sofía. Como se ve en las figuras 4 y 5, los registros de temperatura durante el periodo 2006 en el nivel más bajo de ambos sondeos en el verano es superior a $\operatorname{los} 0^{\circ} \mathrm{C}$, por lo que el espesor de la capa activa es superior a la profundidad del sondeo. Para este mismo año, el periodo de congelación fue de 258 días para Reina Sofía y de 166 días para Incinerador, mostrando ambos su mínima anual el día 25 de Agosto, con $-12,8^{\circ} \mathrm{C}$ y $-10,0^{\circ} \mathrm{C}$ respectivamente.

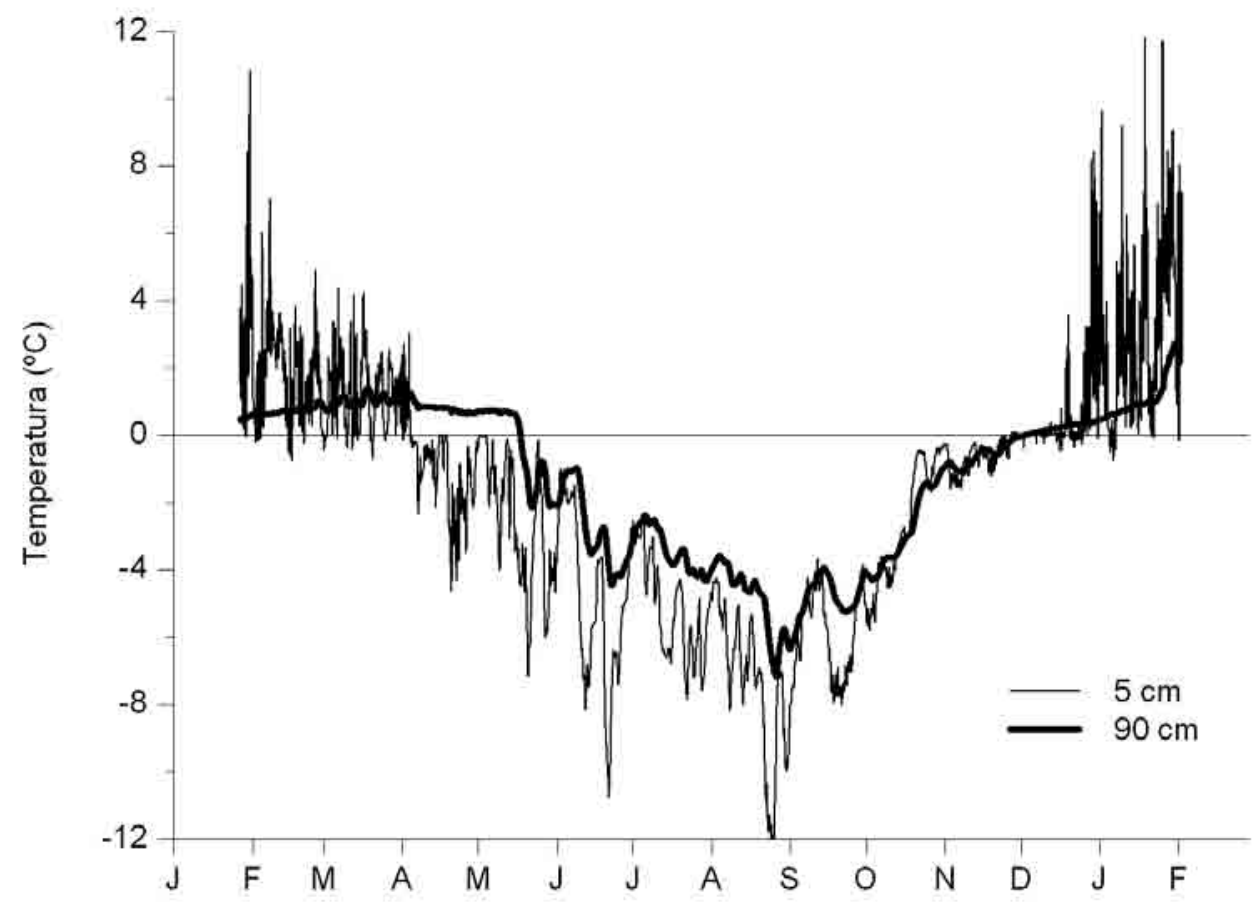

Fig. 4 - Temperaturas de la capa activa en el pico Reina Sofía durante el 2006. Fig. 4 -Active layer temperatures at Reina Sofia peak in 2006. 


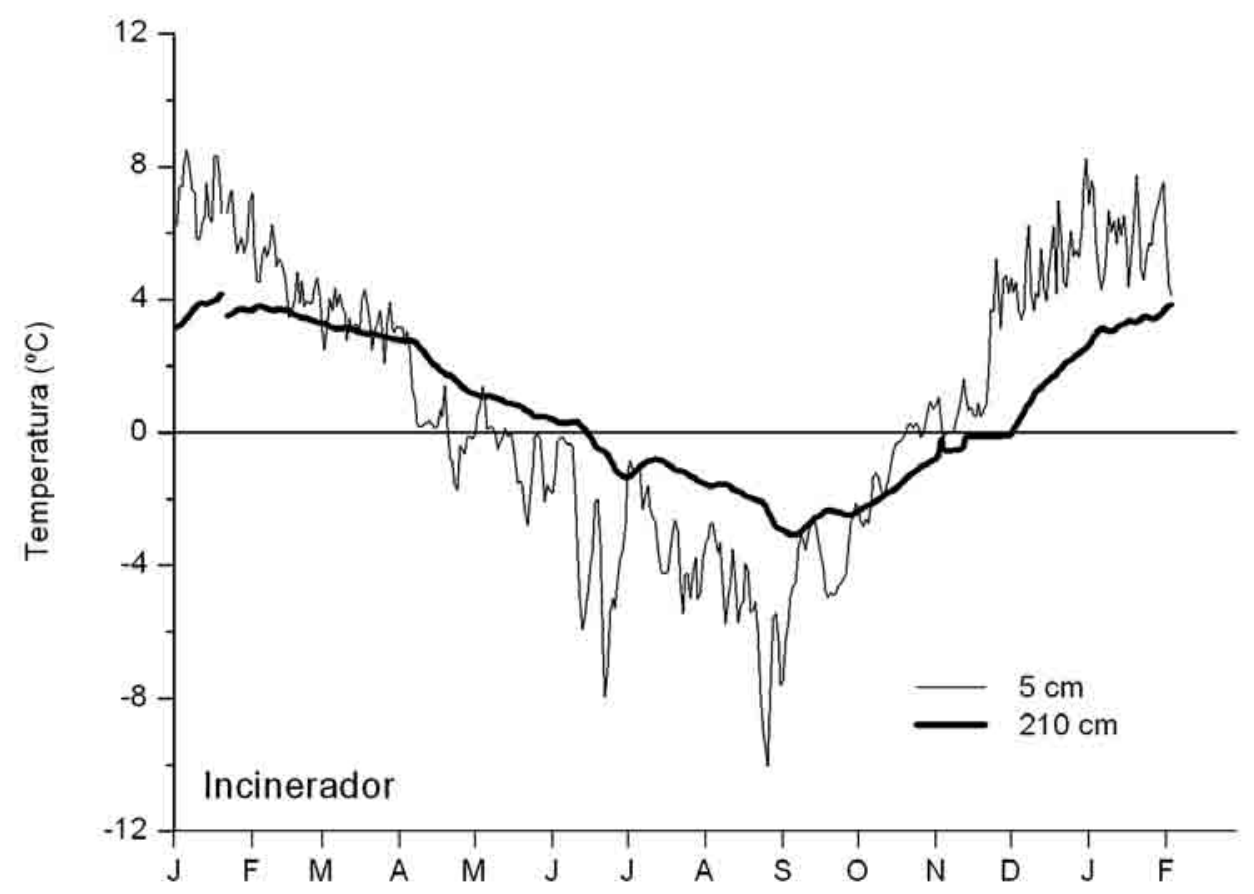

Fig. 5 - Temperaturas de la capa activa en Incinerador durante el 2006.

Fig. 5 - Temperaturas da camada activa no sítio Incinerador em 2006.

Al igual que en Decepción, se instalaron dos puntos de medida TMEM en Punta Polaca y Caleta Española para determinar la microerosión por hielo en la plataforma costera.

\section{CONCLUSIONES}

Las campañas antárticas de 2007 a 2008 realizadas en el cuadro del proyecto Permamodel fueron importantes para el avanze en la instalación de nuevos sitios de monitoreo incluidos dentro de las redes internacionales ANTPAS y TSP del Año Polar Internacional. De particular relieve son los CALM-S instalados en el Pico Reina Sofía y Collado Ramos (Isla Livingston) y las mejoras introducidas en Crater Lake (isla Decepción).

El espesor de capa activa del emplazamiento CALM-S en Crater Lake presento valores entorno a los $35 \mathrm{~cm}$, muy próximos a $\operatorname{los} 32 \mathrm{~cm}$ del año anterior. Estos datos nos hacen plantear para el futuro registrar a lo largo del año el comportamiento térmico del área CALM-S en dos dimensiones, a través de 16 sondeos de entre 40 y $50 \mathrm{~cm}$ de profundidad, para establecer una correlación entre el comportamiento térmico y espesor máximo de capa activa. 
Finalmente, la serie de datos de 7 años en los sondeos de Incinerador y Reina Sofía, es ya suficiente para comenzar un análisis sistemático de estos datos en un cuadro de cambio climático.

\section{BIBLIOGRAFÍA}

Brown J, Hinkel K.M, Nelson F E (2000) The Circumpolar Active Layer Monitoring (CALM) program: research designs and initial results. Polar Geography, 24:165-258

Bockheim J (2004) International Workshop on Antarctic Permafrost and Soils - Final Report. University of Winconsin, Madison, WI.

Burguess M, Smith S, Brown J, Romanovsky V (2001) The Global Terrestrial Network of Permafrost (GNT-P) The status report. IPA Executing Committee Meeting, Rome.

Jones P D, Reid, P A (2001) A data bank of Antarctic surface temperature and pressure data. ORNL/ CDIAC-27 NDP-032. Carbon Dioxide Information Analysis Center, Oak Ridge National Laboratory, U.S. Department of Energy, Oak Ridge, Tennessee. [Accedido el 10 de Marzo de 2008)] http://www.antarctica.ac.uk/climate/surfacetemps/

King J C, Turner J (1997) Antarctic Meteorology and Climatology. Cambridge University Press. Cambridge.

Ling F, Zhang T (2004) A numerical model for surface energy balance and thermal regime of the active layer and permafrost containing unfrozen water, Cold Regions Science and Technology, 38: $1-15$.

López-Martinez J, Vilaplana J M, Martinez De Pisón E, Calvet J, Arché A, Serrat D, Pallas R (1992) Geomorphology of selected areas in Livingston Island, South Shetlands Islands. In J. LópezMartínez (ed.), Geología de la Antártida Occidental. Simposios, T-3, III Congreso Geológico de España, Salamanca: 271-282.

Simonov I M (1977) Physical geographical description of Fildes Peninsula (South Shetland Island). Polar Geography, 35: 145-162.

Styszynska A (2004) The origin of coreless winters in the South Shetlands area (Antarctica). Polish Polar Research, 25: 45-66.

Van Everdingen R (ed.) (1998) Multi-language Glossary of Permafrost and Related Ground-Ice Terms. International Permafrost Association Standing Committee on Data Information and Communication. Circumpolar Active-Layer Permafrost System, Version 2.0. Edited by M. Parsons and T. Zhang. Boulder, CO: National Snow and Ice Data Center/World Data Center for Glaciology. CD-ROM.

Zhang T, Osterkamp T E, Stammes K (1996) Influence of the depth hoar layer of seasonal snow cover on the ground thermal regime. Water Resources Research, 32(7): 2075-2086. 